\title{
1 Homothorax Controls a Binary Rhodopsin Switch in Drosophila
}

\section{Ocelli}

3

4 Abhishek Kumar Mishra ${ }^{*}$, Cornelia Fritsch¹, Roumen Voutev², Richard S. Mann² and Simon G. Sprecher ${ }^{1,3^{*}}$

6

$7{ }^{1}$ Institute of Cell and Developmental Biology, Department of Biology, University of 8 Fribourg, Chemin du Musée 10, 1700, Fribourg, Switzerland, 2Department of

9 Biochemistry and Molecular Biophysics and Neuroscience, Mortimer B. Zukerman

10 Mind Brain Behavior Institute, Columbia University, New York, NY 10027, USA

11 3Lead Contact

*Correspondence: simon.sprecher@gmail.com; abhimishra313@gmail.com

14 Keywords: Drosophila, Photoreceptor, Homothorax, Ocelli, Compound eye,

15 Rhodopsin, Gene duplication, Evolution, cell fate

18 Running title: Hth regulates binary Rhodopsin switch in Drosophila ocelli 


\section{Abstract}

23 Visual perception of the environment is mediated by specialized photoreceptor (PR) neurons of the eye. Each PR expresses photosensitive opsins, which are activated by a particular wavelength of light. In most insects, the visual system comprises a pair of compound eyes that are mainly associated with motion detection, color or polarized light perception and a triplet of ocelli that are thought to be critical during flight to detect horizon and movements. It is widely believed that evolutionary diversification of compound eye and ocelli in insects occurred from an ancestral visual organ around 500 million years ago. Concurrently, opsin genes were also duplicated to provide distinct spectral sensitivities to different PRs of compound eye and ocelli. In the fruit fly Drosophila melanogaster, Rhodopsin1 (Rh1) and Rh2 are closely related opsins that are originated from the duplication of a single ancestral gene. However, in the visual organs, Rh2 is uniquely expressed in ocelli whereas Rh1 is uniquely expressed in outer PRs of the compound eye. It is currently unknown how this differential expression of Rh1 and Rh2 in the two visual organs is controlled to provide unique spectral sensitivities to ocelli and compound eyes. Here, we show that Homothorax (Hth) is expressed in ocelli and confers proper rhodopsin expression. We find that $\mathrm{Hth}$ controls a binary Rhodopsin switch in ocelli to promote Rh2 expression and repress Rh1 expression. Genetic and molecular analysis of $r h 1$ and $r h 2$ supports that Hth acts through their promoters to regulate Rhodopsin expression in the ocelli. Finally, we also show that when ectopically expressed in the retina, hth is sufficient to induce $\mathrm{Rh} 2$ expression only at the outer PRs in a cell autonomous manner. We therefore propose that the diversification of rhodpsins in the ocelli and retinal outer PRs occurred by duplication of an ancestral gene, which is under the control of Homothorax. 

in nature is essential for many aspects of life. Animals rely heavily on visual cues to perform complex tasks such as navigation to find food, mates and shelter as well as social interactions. Visual cues are perceived by visual organs that contain photoreceptors (PRs) as light-sensing structures. PRs are specialized cells that gather information from the surrounding world which is subsequently processed by the brain. Each PR expresses a unique photosensitive opsin/rhodopsin and they define the wavelength of light by which a PR will be activated.

It is believed that eyes have evolved separately due to fundamental differences between visual organs of different animals [1]. However, it is also known that eye development in different animal phyla share a common genetic network initiated by Pax6 gene orthologs [2]. Finding similarities in the gene regulatory network that controls eye development further strengthened the idea that phylogenetically diverse eye types may share a conserved eye developmental program [3-5]. Insects are among the largest and most diverse animal groups. Decoding eye development in insects offers great opportunity to unravel developmental insights that lead to the emergence of evolutionary complexity.

In most insects, compound eyes represent the prominent visual organ that is responsible for providing major share of the visual information. In the fruit fly Drosophila melanogaster, each compound eye consists of approximately 850 ommatidia and each ommatidium houses eight PRs: six outer PRs and two inner PRs [6]. Additionally, winged insects (such as Drosophila) possess ocelli that are comparatively simple photosensory organs embedded in the dorsal head cuticle [7]. In Drosophila, a triplet of ocelli (one medial and two lateral) are arranged in a triangular shape between the two compound eyes and the dorsal vertex of the head [8]. It is 
72 believed that insect compound eye- and ocellus-like precursor structures have segregated from an ancestral eye over 500 million years ago [9, 10]. The evolution of new opsin genes by gene duplication enabled these visual organs to perform different functions that require distinct spectral sensitivities [11]. In Drosophila, phylogenetic analysis supports that Rhodopsin1 (Rh1), Rh2 and Rh6 have originated from a common ancestral gene [12]. A first gene duplication may have separated Rh6 from Rh1/Rh2 and a second gene duplication may have separated the closely related Rh1 and Rh2 [12]. The green sensitive Rh6 is expressed in the inner PRs of the compound eye and is critically involved in color perception [13-15]. The blue-green sensitive Rh1 is expressed in the outer PRs of the compound eye and is mainly associated with motion detection [16-18]. Conversely, the UV-blue sensitive Rh2 is expressed in PRs of the ocelli [19-21] and is proposed to be involved in horizon sensing and flight stabilization [22, 23]. While it is known that $r h 1, r h 2$ and $r h 6$ are genetically linked [24], it is still unknown how they are differentially expressed in different PRs in Drosophila. Here we show that the homeodomain transcription factor Homothorax $(\mathrm{Hth})$ regulates $\mathrm{Rh} 2$ expression in the ocelli. We demonstrate that $\mathrm{Hth}$ is expressed in ocellar PRs and controls a binary rhodopsin switch by promoting Rh2 expression and repressing Rh1 expression in ocelli. We also demonstrate that misexpression of $\mathrm{Hth}$ forces outer PR of the retina to induce Rh2 expression and clonal expression in the

91 retina suggest that this process is cell autonomous. Furthermore, genetic and molecular analysis of $r h 1$ and rh2 shows that the rhodopsin switch in ocelli is transcriptionally controlled by $\mathrm{Hth}$ and that it may act directly through $r h 1$ and $r h 2$ promoter sequences. Finally, we argue that while Hth maintains Rh3 fate in the DRA [25], it initiates Rh2 fate in the ocelli. The results presented here greatly adds to our 

made available under aCC-BY 4.0 International license.

96 understanding of how genetically linked opsins are spatiotemporally controlled to

97 provide distinct spectral sensitivity to different visual organs.

98

99 
Differential expression of rhodopsins in the compound eye and ocelli

It is believed that the hexapod ancestor of extant insects only had a single visual

eye and ocelli) evolved as a result of a morphological bifurcation event over 500 million

years ago $[10,11]$ (Figure $1 \mathrm{~A}$ and $1 \mathrm{C}$ ). It is hypothesised that different functions of compound eye and ocelli in conjunction of gene duplication events of opsins led to the emergence of an ocelli specific rhodopsin gene, with a distinct spectral sensitivity [11]. In the Drosophila compound eye, brightness detection is achieved by inputs from six outer-PRs (R1-R6) that express the long wavelength opsin Rhodopsin 1 (Rh1).

Drosophila uses outer PRs mainly for motion detection and dim light vision [16-18].

Color vision requires two inner PRs that encode either one of the UV-sensing opsins

112 Rh3 or Rh4 in R7 PRs, and either the blue-sensing Rh5 or the green-sensing Rh6 in R8 PRs [26, 27]. In most insects, ocelli express an opsin, which is different from those present in the compound eye $[11,21,28]$. In Drosophila, PRs of the ocelli express the long wavelength opsin Rh2 (Figure 1B) that is sensitive to the violet spectrum of wavelengths of light [19-21]. The different spectral sensitivity of ocelli is also reflected by their involvement to perform different functions than the compound eye and they are believed to detect horizon, control head orientation and stabilize flight posture while flying [22]. To get an insight of rhodopsin gene duplications in Drosophila, we compared the coding sequences of rh1 to rh6 by generating a phylogenetic tree (by using MUSCLE online tool; phylogeny.fr) [29]. The phylogenetic tree made by maximum likelihood method showed two clades with branching support value of 1 each (Figure 1D). Clade-I showed a tandem gene duplication that separated Rh5 from

124 the closely related $\mathrm{Rh} 3 / \mathrm{Rh} 4$. Clade-II represents long-wavelength opsin gene 
duplications in Drosophila where first a tandem gene duplication separated Rh6 from Rh1/Rh2 (Figure 1D). Rh6 subsequently got expressed in the inner PRs of the compound eye (Figure 1J). A further gene duplication led to the separation of Rh1 and Rh2 (Figure 1D). While Rh1 subsequently got expressed in the outer PRs of the compound eye (Figure 1H), Rh2 got exclusively expressed in the ocelli (Figure 1F). To analyse the evolutionary origin of long-wavelength opsin gene duplications, we generated a phylogenetic tree by using amino acid sequences of Drosophila opsins and probable Rh1, Rh2 and Rh6 orthologs from other dipteran species (Ceratitis capitata or med fly, Musca domestica or house fly, Glossina palpalis or tsetse fly, Lucilia cuprina or Australian sheep blow fly and Aedes aegypti/Anopheles gambiae or mosquitoes; sequences collected from Feuda lab on bitbucket $\begin{array}{llll}\text { (https://bitbucket.org/Feuda-lab/opsin diptera/src/master/) } & \text { [30]. The resulting }\end{array}$ phylogenetic alignment of Rh1, Rh2 and Rh6 suggests that Rh6 is ancestral to all dipteran species including mosquitoes. A common Rh1/Rh2 ortholog originated from a duplication of Rh6 in the lineage leading to the higher dipterans and a second gene duplication separated Rh1 from Rh2. Further gene duplications of Rh6 occurred in the mosquitoes and of Rh1 in the house fly Musca domestica (Figure S1).

\section{Homothorax regulates a binary Rhodopsin switch in the ocelli}

In the compound eye, the homeodomain transcription factor Homothorax (Hth) is expressed in the dorsal rim area (DRA) of inner PRs, where it has been shown to be both necessary and sufficient to regulate Rh3 expression and thereby critically contributes to the polarized-light sensing system $[25,31]$. We found that $H$ th is also expressed in all PRs of the ocelli (Figure 2A). Since ocellar PRs express Rh2, we first analysed if $\mathrm{H}$ th is involved in regulating this expression. We performed knockdown of 
150

151

152

153

154

155

156

157

158

159

160

161

162

163

164

165

166

167

168

169

170

171

172

173

174

hth using the pan-photoreceptor driver IGMR-Gal4 and observed a complete loss of

Rh2 expression in PRs of the ocelli (Figure 2B). Additionally, we also observed that in $h$ th knockdown, the loss of $\mathrm{Rh} 2$ expression in the ocelli is compensated by gain of $\mathrm{Rh} 1$ (Figure 2B), a Rhodopsin that is normally expressed in outer PRs of the compound eye. Thus, Hth regulates a binary switch of Rhodopsin in the ocelli where it promotes Rh2 expression and represses Rh1 expression.

Hth often acts together with Extradenticle (Exd) [32, 33]. Therefore. to see if Exd is also involved to regulate the binary Rhodopsin switch in the ocelli, we performed knockdown of exd with IGMR-Gal4 and found that it also resulted in the loss of Rh2 expression and gain of Rh1 expression in the ocellar PRs (Figure 2C).

In the compound eye, a feedback mechanism has been described that allows Rh6 to transcriptionally repress Rh5 in yellow R8 PRs [34]. Since in hth knockdown, ectopic Rh1 expression in the ocelli replaces Rh2, we speculated that Hth may repress Rh1 in order to allow Rh2 expression. Knockdown of $h$ th in turn would remove this repression and as a result Rh1 would get activated and would repress Rh2 in the ocelli. To check this hypothesis, we misexpressed Rh1 in ocelli by using IGMR-Gal4 and found that ectopic expression of Rh1 was not sufficient to repress Rh2 and in this case ocelli co-expressed both Rh1 and Rh2 (Figure S2A). Thus, loss of Rh2 in hth knockdown seems to be independent of Rh1 repression.

To further test whether switching Rhodopsin expression might also alter PR identity based on other molecular markers of outer PRs in the retina, we monitored Seven-up (svp) and BarH1 expression in the ocelli in hth knockdown. Svp is a steroid hormone receptor and is expressed in $\mathrm{R} 3 / \mathrm{R} 4$ and $\mathrm{R} 1 / \mathrm{R} 6$ pairs whereas $\mathrm{BarH} 1$ is a homeobox transcription factor expressed in the R1/R6 pair of the developing compound eye $[35,36]$. We found that neither Svp nor BarH1 are expressed in 
wildtype nor in hth knockdown ocelli (Figure S3A, S3B, S3C and S3D). Therefore, although ocellar PRs gained Rh1 and lost Rh2 expression when hth was knocked down, they do not seem to undergo an identity change towards retinal outer PRs.

We next investigated whether regulation of this Rhodopsin switch by Hth occurs at the transcriptional level by using ocelli specific rh2-lacZ [20] and outer PR specific rh1-lacZ [36] reporter lines. In wildtype control animals, $\beta$-gal expression was specifically observed in ocelli in case of rh2-lacZ (Figure 2D) whereas no expression was observed in ocelli in the case of rh1-lacZ (Figure 2G). In hth knockdown background, we found that $\beta$-gal expression from rh2-lacZ is completely abolished from the ocelli (Figure 2E), whereas ectopic $\beta$-gal expression from rh1-lac $Z$ is now seen in ocellar PRs (Figure $2 \mathrm{H}$ ). Therefore, $\mathrm{H}$ th is indeed involved in regulating a binary Rhodopsin switch in the ocelli by promoting transcription of $r$ h2 and repressing transcription of $r h 1$.

Since Hth and Exd act together, we next investigated whether Exd is also involved in regulating $r h 1$ and $r h 2$ transcriptionally. We indeed found that $\beta$-gal expression from rh2-lacZ was lost (Figure 2F) and ectopic $\beta$-gal expression from rh1lacZ (Figure 2I) was observed in exd knockdown background in ocellar PRs.

We have previously shown that the homeodomain transcription factor Hazy (Flybase: Pph13 for Pvull-Pstl homology 13) controls expression of Rh2 in the ocelli $[37,38]$. We therefore next asked if Hazy and $\mathrm{Hth}$ act jointly to regulate the Rhodopsin switch in the ocelli. However, we find that in hazy/- null mutant flies, absence of Rh2 expression is not accompanied with the ectopic expression of Rh1 in ocellar PRs (Figure S2B). Moreover, we found that $\mathrm{Hth}$ is still expressed in the ocelli of hazy/mutant flies (Figure S2C). Also, the expression of Hazy remained unchanged in ocellar 
199 PRs when knocking down hth (Figure S2D). Thus, the Hth-dependent Rhodopsin switch in the ocelli does not depend on Hazy.

201

Hth controls a binary Rhodopsin switch in ocelli by acting through the

\section{promoters of $\boldsymbol{r h 1}$ and $\boldsymbol{r h} 2$}

Since Hth encodes a homeodomain transcription factor we next investigated if

Hth may act by directly regulating the rhodopsin promotors. Minimal promoter

Drosophila rhodopsin promoters, they are rather short (300-400 bp) and contain a

Rhodopsin-Conserved-Sequence-I (RCSI) element that provides binding sites for

Pax6 orthologs and other factors that promote photoreceptor-specific expression [39].

We aligned the minimal promoter sequences of twelve different Drosophila species of the two promoters was not possible since they have no sequence similarities apart from the RCSI site. A portion of the rh2 minimal promoter sequence overlaps with the coding sequence of the neighbouring gene CG14297 (Figure S5). We identified three potential Hth binding sites in the minimal promoter region of $r h 2(-293 /+55)$ [20]: one within the coding sequence of CG14297, one directly following its Stop codon and one within its 3'UTR. While the sites in the coding sequence and in the 3'UTR are conserved, the site at the Stop codon is only present in the four closest relatives of Drosophila melanogaster. The rh1 minimal promoter region $(-247 /+73)$ [36] contains two potential Hth binding sites upstream of the RCSI (Figure S4). To test if the Hth binding sites in the $r h 1$ and $r h 2$ promoter regions may be involved in the Rhodopsin switch in ocelli, we created transgenic flies containing transgenes with the minimal 
promoter regions of $r h 1$ or $r h 2$ driving GFP ( $r h 1-G F P$ and $r h 2-G F P$ [37]). Next, in order to abolish Hth binding, we introduced point mutations in the Hth binding regions of the rh1 and rh2 promoters and created rh1(hth mut)-GFP and rh2(hth mut)-GFP transgenic flies (Figure 3A and 3D) (See material and methods for details). In support with the previous observations, we find rh2-GFP expression in the ocelli (Figure 3B) whereas rh1-GFP is not expressed in ocellar PRs (Figure 3E). However, by mutating the Hth binding sites in the promoter region of $r h 2$ (Rh2 (hth mut)-GFP), we observed the Hth binding sites in the promoter region of $r h 1$ ( $r h 1$ (hth mut)-GFP) leads to ectopic GFP expression in the ocelli (Figure 3F).

\section{Hth is sufficient to induce Rh2 expression in the outer PRs of the retina} expression and ectopic expression of $\mathrm{Hth}$ under the control of IGMR-Gal4 was sufficient to block the expression of inner PR Rhodopsins (Rh4, Rh5 and Rh6) and to induce Rh3 expression in all inner PRs. However, in outer PRs, expression of Rh1 was not affected suggesting that only inner PRs were responsive to $\mathrm{Hth}$ misexpression [25]. We next investigated if misexpression of $\mathrm{Hth}$ in the retina was also sufficient to induce Rh2 expression. In the wildtype retina, Rh1 was uniquely expressed in outer PRs whereas Rh2 was absent (Figure 4A, 4A' and 4A"). When Hth was misexpressed

244 in the retina under the control of IGMR-Gal4, we observed that Rh2 was now ectopically co-expressed in all outer PRs. However, we found that Rh1 expression was unaffected and it was still expressed in all outer PRs (Figure 4B, 4B' and 4B'). 
inner PRs) suggesting that only Rh1 expressing PRs were competent to induce Rh2 expression in the retina, while inner PRs were not. (material and methods for details). Flip-out clones were marked by presence of GFP

expression. We found that when clones were generated in the inner PRs, Rh2

expressing clones (Figure 5B, 5B' and 5B"). Thus, ectopic Rh2 expression in Rh1

expressing PRs of the retina is a cell-autonomous process. Expression of Hth in inner

PR clones did not have any effect on Rh1 expression in the neighbouring outer PRs

(Figure 5C, 5C' and 5C') and Rh1 expression was maintained when clones were generated in the outer PRs (Figure 5D, 5D' and 5D').

\section{Role of Scrib and Ets65A as potential repressors of Rh1 in the ocelli.}

To understand if Hth acts together with any of its known interactors to regulate

Rh1 and Rh2 expression in the ocelli, we performed a knockdown mini-screen of for Rh1 and Rh2 expression in the ocellar PRs. In agreement with the previous finding,

267 Rh2 expression was lost in the ocelli in calmodulin-binding transcriptional activator (camta), longitudenals lacking (lola) and defective proventriculus (dve) knockdown (Mishra et al., 2016). However, we did not observe a gain of Rh1 in any of these knockdowns (Figure 6B, 6C and 6D). Surprisingly, in the mini-screen, we found that

271 knockdown of the scaffolding protein Scribble (Scrib) $[41,42]$ and the Ets domain 
transcription factor Ets65A $[43,44]$ resulted in a gain of Rh1 expression in the ocellar PRs without changing Rh2 expression (Figure 6E and 6F).

Taken together, we showed that Hth regulates a binary Rhodopsin switch in ocelli by promoting Rh2 expression at the cost of Rh1. We found that regulation of $\mathrm{Rh} 1$ and $\mathrm{Rh} 2$ is transcriptionally controlled by $\mathrm{Hth}$ and it acts together with its binding partner Exd. We also found that Hth regulates the Rhodopsin switch by acting through the promoters of $r h 1$ and $r h 2$. We further demonstrated that misexpression of $H$ th in the retina modifies outer PRs to acquire Rh2 expression resulting in outer PRs coexpressing Rh1 and Rh2 and we show that this process is cell-autonomous Finally, by knockdown mini-screen, we identified Scrib and Ets65A as potential repressors of Rh1 expression in the ocelli (Figure 7).

\section{Discussion}

\section{Homothorax expression provides new insights to understand Rhodopsin fate in}

\section{Drosophila}

In insects, ocelli represent a fundamentally simpler visual organ whose spectral sensitivity is different from the compound eye. The unique spectral sensitivity of ocellar PRs in Drosophila is provided by the violet-sensing Rh2 [21]. The presence of this particular Rhodopsin exclusively in ocelli and not in the retina may explain why ocelli perform different functions than the compound eye. We have characterized roles of $\mathrm{Hth}$ during terminal differentiation of ocellar PRs and showed that $\mathrm{Hth}$ acts together with Exd and regulates a binary Rhodopsin switch in ocelli that promotes Rh2 expression and represses Rh1 expression. Hth is known to be expressed in the ocellar primordium of the early third instar laval (L3) eye antennal imaginal disc but gets downregulated later at mid- to late-L3 [45]. However, it is unknown how expression of 
Hth is re-induced in ocellar PRs. One possible hypothesis could be that a temporal change during metamorphosis such as a pulse of ecdysone hormone with additional signals induces Hth expression in ocelli. In our study, we show that Hth is expressed in all mature and terminally differentiated PRs of ocelli.

Hth performs similar functions in PRs of the ocelli and the DRA of the retina: in ocelli, it induces Rh2 expression by repressing Rh1 whereas in the DRA of the retina it induces Rh3 by repressing inner-PR Rhodopsins [25]. Loss of $h$ th transforms the DRA into odd-coupled ommatidia where $\mathrm{Rh} 3$ is expressed in $\mathrm{R} 7$ and Rh6 in $\mathrm{R} 8$ suggesting that the Rh3/Rh6 pair represents the default state of Rhodopsins in inner PRs of the retina [25]. In ocelli, we find ectopic expression of Rh1 by loss of $h$ th suggesting that Rh1 may be the default state in ocellar PRs. Loss of Hth leads to ectopic expression of the R8 specific transcription factor Senseless in the DRA, which is otherwise not expressed in the wildtype DRA suggesting a fate change of the DRA to become odd-coupled ommatidia [31]. Interestingly, $\mathrm{H}$ th loss in ocelli does not induce expression of outer PR-specific transcription factors (such as Svp and BarH1) suggesting that the ocellar PR fate may not have been changed.

\section{Genetic and molecular analysis of Hth in the ocelli during PR development}

The DNA binding property of $\mathrm{Hth}$ is required for proper DRA fate in the retina [31]. Similarly, we observed that Hth regulates a binary Rhodopsin switch in ocelli by transcriptionally controlling $r h 1$ and $r h 2$ expression through binding to their upstream DNA sequences. The homeodomain transcription factor Hazy controls Rhodopsin expression in the retina and in ocelli $[37,38]$. However, epistatic analysis showed that both Hth and Hazy act independently to control Rh2 expression in ocelli. Misexpression of $h$ th in all developing PRs of the retina is sufficient to induce a fate 
switch where inner PRs are transformed into PRs of DRA [31]. Rh3 is expanded to all inner PRs whereas specific loss of Rh4, Rh5 and Rh6 was seen. However, outer PR fate was not changed suggesting that only those PRs, which were previously committed to become inner PRs during development, were responsive to $\mathrm{Hth}$ [31]. We additionally found that outer PRs, too were responsive to $h$ th misexpression since they induce Rh2 expression while maintaining its default fate by expressing Rh1. However, the genetic program activated by Hth misexpression to induce $\mathrm{Rh} 2$ expression in outer PRs is still unknown. One hypothesis could be that Hth directly activates Rh2 expression in the ocelli but it requires an additional cofactor to repress Rh1 expression.

This cofactor could be present in the ocelli but not in the retina and this would explain why knockdown of $\mathrm{Hth}$ activates $\mathrm{Rh} 1$ expression in the ocelli but is not sufficient alone to repress Rh1 expression in the retina. We additionally found that knockdown of Scrib and Ets65A induces Rh1 expression in the ocelli. However, further experiments will be required to establish the role of Scrib and Ets65A as potential repressors of Rh1 expression in ocelli versus retina.

\section{Rhodopsin duplication and role of Hth during ocelli diversification.}

Photoreception is achieved by expression of different opsins in conjunction with an array of proteins of the phototransduction cascade. Each PR contain a specific opsin, which are light sensing proteins that define the particular wavelength of light to which a given PR will respond. In most insect species, opsins were mainly categorized into three major clades: 1) UV-sensitive opsins 2) blue-sensitive opsins and 3) longwavelength (LW) opsins, which are ranging from violet such as Rh2 in Drosophila [46] to green-red in some butterflies [47]. Presence of all three opsin types in most insect species may imply that the ancient retina was trichromatic [48]. Further phylogenetic 
analyses support ancestral trichromacy in insects and show that opsin sensitivity must have derived from a single ancestral opsin as a result of gene duplications [12]. In many insect species, spectral sensitivity of opsin gene clades is represented by more than one paralog such as the long wavelength opsin clade consisting of three paralogs in Drosophila (Rh1, Rh2 and Rh6), five in the mosquito Anopheles gambiae and two in the honeybee Apis mellifera [12]. It is believed that a first tandem gene duplication in Drosophila separated Rh6 from its sister paralog Rh1/Rh2 and while Rh6 is retained in the inner PRs of compound eye, Rh1/Rh2 was associated with the outer PRs of retina and PRs of ocelli. A second gene duplication event led to the diversification of Rh1 and Rh2 by accumulation of further amino acid changes in their sequence. While Rh1 expression was retained in the outer PRs of the compound eye, Rh2 became associated with the PRs of the ocelli. Our results also suggest that the ability of Hth to bind to both promoters may have been a prerequisite during evolution to differentially regulate the expression of the two paralogs and thus, provide spectral sensitivity of ocelli by promoting Rh2 expression and repressing Rh1 expression. However, it would be interesting to know if a change in the spectral sensitivity of ocelli upon Hth loss would in turn change their specific functions. Also, if gene duplications of opsins occurred during evolution to allow the diversification of different spectral sensitivities in different visual organs, it would be interesting to know what kind of spectral sensitivity was present in the ancestral visual organ. We conclude that $H$ th may act as an evolutionary factor required by ocelli to provide their unique spectral identity to ocellar PRs that are marked by expression of Rh2. To maintain this unique spectral identity, Hth controls a binary Rhodopsin switch to repress outer PR fate in ocelli by repressing Rh1 expression. 
372 The opposing regulatory function of Hth on its direct targets.

373 We show here that Hth acts along with its binding partner Extradenticle to function

374 both as a transcriptional activator of $r h 2$ and as a transcriptional repressor of $r h 1$.

375 Usually, Hth in conjunction with its binding partner Extradenticle acts as a

376 transcriptional activator. Interestingly, by knocking down exd, we also observed a lack

377 of Rh2 and a gain of Rh1 expression suggesting that both acts together. It was

378 previously shown that binding of $\mathrm{Hth}$ to the DNA requires the presence of the co-factor

379 Exd $[49,50]$. Therefore, likely regulation of Rh1 and Rh2 depend on the presence of

380 both $\mathrm{Hth}$ and Exd binding to the regulatory region. Hth has also been shown to function

381 in gene repression [51]. In this case the formation of the repressor complex occurs

382 directly at the regulatory regions of the repressed gene and depends on the proximity

383 of DNA-binding sites for different components of the complex in the regulatory region.

384 The same mechanism could also explain the opposing regulatory effect that Hth has on rh1 in comparison to rh2 in the ocelli. Analysis of transcription profiles of different PR types would provide a list of Hth interactors expressed in the ocelli versus the outer PRs of the retina. Binding analysis of these interactors to the $r h 1$ and rh2 minimal promoter sequences would help to identify the potential repressor complex forming on the $r h 1$ promoter, helping to better understand the regulatory functions of $\mathrm{Hth}$.

\section{Author contributions}

AKM and SGS designed all experiments. AKM, CF and RV performed experiments and generated reagents/materials. AKM, CF, RV, RSM and SGS wrote the manuscript. 
397 We thank C. Desplan, J. Rister, A. Salzberg, T. Cook, D. Vasiliauskas, Developmental

398 Studies Hybridoma Bank (DSHB) and Bloomington Stock Center for providing flies and antibodies. We thank UniFr bioimage team for maintaining imaging facility and all members of Egger and Sprecher lab for fruitful discussions. This work is supported by 401 the Swiss National Science foundation grant number 310030_188471 to SGS, the 402 Novartis foundation for biomedical research grant number 18A017 to SGS and the 403 National Institute of Health grant number 5R35GM118336-05 to RSM. The funders had no role in study design, data collection and analysis, decision to publish, or preparation of the manuscript.

407 Materials and methods

\section{Fly stocks}

409 Wildtype Canton S flies have been used in this study. Other fly strains used were:

410 UAS-hth ${ }^{R N A i}$ (BL27655 and BL34637; we do see the Rhodopsin switch phenotype in

411 both RNAi lines. However, all the experiments were done in BL27655), UAS-exd ${ }^{R N A i}$

412 (BL29338 and BL34897; Rhodopsin switch phenotype was observed in both RNAi 413 lines. However, all experiments were done in BL34897). For knockdown mini-screen, we used UAS-RNAi flies from Bloomington's Drosophila stock center and the stock numbers are mentioned in Figure S6. Other fly strains are: UAS-rh1 [34], UAS-hth [52], rh1(-252/+57)-lacZ[36], rh2 (-309/+32)-lacZ [20], rh2 (-293/+55)-GFP [37], hazy

417 '-[53], hsFLP; UAS-mCD8::GFP; IGMR<wt>Gal4 (a gift from Claude Desplan lab). The 418 following transgenic lines were made in this study: rh2 (hth mut)-GFP, rh1-GFP and 419 rh1 (hth mut)-GFP. Flies were reared on standard food medium and at $25^{\circ} \mathrm{C}$. 420 Knockdown flies were grown at $29^{\circ} \mathrm{C}$ temperature. For $h$ th overexpression clones by 421 "flip-out" technique, flies were grown at $25^{\circ} \mathrm{C}$ and heat shock at $37^{\circ} \mathrm{C}$ was performed 
422 for half an hour at the late pupal stage. Vials were placed back at $25^{\circ} \mathrm{C}$ and freshly

423 hatched flies were dissected.

424

425 Generation of $r h 1$ and $r h 2$ reporter transgenes

426 The rh1 minimal promoter $(-247$ to +73$)$ was PCR amplified from genomic DNA with

427 primers "rh1 enh Kpn fw" (gcggtacCTGGAGACTCAAGAATAATACTCGGCCAG) and

428 "rh1 enh Xba re" (gatctagAGGGTTCCTGGATTCTGAATATTTCACTG) and cloned

429 into pBluescript vector using the $\mathrm{Kpnl}$ and Xbal sites added to the primers. For cloning

430 of the $r h 2$ minimal promoter see [37]. The same $r h 1$ and $r h 2$ promoter fragments were

431 in vitro synthesized (BioCat) altering the sequences of the potential Hth binding sites.

432 The first $\mathrm{Hth}$ site in the $r h 1$ promoter (TGACAT) was changed to TaAgcT creating a

433 Hindlll restriction site. The second Hth site in the rh1 promoter (CTGTCG) was

434 changed to CTaaaG. The first Hth site in the rh2 promoter (GGACAG) was changed

435 to GtttAG, the second Hth site in the rh2 promoter (GTGTCA) was changed into

436 agcTgA and the third Hth site (CTGTCC) was changed to CTaaaC. Both versions of

437 the two enhancers were cloned into a GFP reporter plasmid containing eGFP, a

438 miniwhite marker and an attB site kindly provided by Jens Rister. The plasmids were

439 injected into nos- $\varphi \mathrm{C} 31$; attP40 flies for integration on the second choromosome using

440 the $\varphi$ C31 site-specific integration system [54].

442 Immunohistochemistry

443 Adult ocelli were dissected and stained by a protocol published in [37] whereas

444 dissection and immunohistochemistry of adult retinas were done according to [55].

445 After the immunostainings, tissue samples were mounted by using Vectashield $\mathrm{H}$ -

4461000 (Vector laboratories). Primary antibodies and their dilutions were as follows: 
447 Rabbit anti-Rh2 1:100 [37], Rabbit anti-Rh6 1:10,000 [56], Rabbit anti-Hth 1:500 [57],

448 Chicken anti-ßGal 1:1000 (Abcam), Chicken anti-GFP 1:2000 (Life technologies),

449 Rabbit anti-Hazy 1:500 [53], Mouse anti-Svp 1:100 [58], Rat anti-BarH1 1:200 [59],

450 Mouse anti-Rh1 1:20, Rat anti-Elav 1:20 and Mouse anti-Chp 1:20 (Developmental

451 Studies Hybridoma bank). Phalloidin conjugated with Alexa-568 and Alexa-647

452 (Sigma-Aldrich, Life Technologies) marks F-actin and were used (1:1000) during

453 incubation with secondary antibodies. The following secondary antibodies were used:

454 Goat anti-rabbit, Goat anti-mouse, Goat anti-rat and Goat anti-chicken that are 455 conjugated with Alexa-488, Alexa-555 and Alexa-647 (Jackson Immunoresearch). All 456 secondary antibodies are used at 1:200 dilution.

457

458 Confocal microscopy and Image analysis

459 Tissue samples were imaged with a Leica TCS SP5 confocal microscope at a 460 resolution of $1024 \times 1024$ pixels and optical sections were taken in the range of $1-2 \mu \mathrm{m}$ 461 depending on the sample size. Images were further processed and analysed in 462 Fiji/lmageJ and Adobe photoshop 2020 software.

463

464 Figure legends

465 Figure 1: Overview of an insect visual system, its evolutionary diversification 466 and comparison of Rhodopsin expression in the Drosophila ocelli and

467 compound eye. (A) Dorsal view of the head of a fruit fly showing locations of 468 compound eye (CE) and ocelli (black circle). (B) Cross section of a Drosophila adult 469 brain where ocelli are marked by expression of Rh2 (in green). Chp expression (in 470 blue) marked ocellar PRs and Elav expression (in red) marked neuronal population 471 (C) Schematic representation of a morphological bifurcation event that occurred over 
500 million years ago (mya) in an ancestral eye of hexapod ancestors that is believed to form ocelli and compound eye. (D) Schematic representation of gene duplications in Drosophila rhodopsins during evolution. Blue dotted square box marks the long wavelength opsin clade consisting of Rh1, Rh2 and Rh6. It is believed that a first tandem gene duplication separated Rh6 from Rh1/Rh2 and a second gene duplication separated the closely related Rh1 and Rh2. (E-G) Longitudinal sections through the ocelli stained for Rh1 (E), Rh2 (F) or Rh6 (G) shown in green. These proteins, if expressed, are located to the rhabdomeres directly below the lenses, while Elav (red counterstain), which was used to identify the position of the ocelli, localizes to the nuclei of the photoreceptors, which are positioned below the rhabdomeres (compare with schematic drawing in $\mathrm{C})$. $(\mathrm{H}-\mathrm{J})$ Cross sections of photoreceptors in the retina stained for Rh1 (H), Rh2 (I) or Rh6 (J) shown in green and for Phalloidin localising to the rhabdomeres shown in red. $(E, H)$ Rh1 is exclusively expressed in the outer PRs of the retina but not in ocelli. (F, I) Rh2 is uniquely expressed in all ocellar PRs but not in the retina. $(G, J)$ Rh6 is normally expressed in the inner PRs in the retina but not in ocelli.

Figure 2: Hth expression and phenotype in ocelli. (A) Antibody staining of $H$ th in the wildtype ocelli showing its expression in all ocellar PRs. Chp (green) and Elav (blue) marks ocellar PR neurons. (B) hth knockdown (IGMR>UAS- $\left.h t h^{R N A i}\right)$ in the ocelli by pan-photoreceptor IGMR-Gal4. In hth knockdown, Rh2 expression (green) is lost and there was a gain of Rh1 expression (red) in the ocellar PRs. (C) exd knockdown (IGMR>UAS-exd ${ }^{R N A}$ ) in ocelli showed a similar phenotype i.e., loss of Rh2 expression (green) and gain of Rh1 expression (red). (D, E, F) Antibody staining of $\beta$ Galactosidase ( $\beta$-Gal) in the control (rh2-lacZ; IGMR/t), in hth knockdown (rh2-lacZ; 
IGMR $>h t h^{R N A i}$ ) and in exd knockdown ocelli. $\beta$ Gal (green) expression was seen in

were marked by Rh1 (red) (E, F). (G, H, I) Antibody staining of $\beta$ Gal in the control (rh1lacZ; IGMR/+), in hth knockdown (rh1-lacZ; IGMR>hth ${ }^{R N A I}$ ) and in exd knockdown ocelli. In the control, ocelli were marked by Rh2 (blue) and $\beta$ Gal (green) expression was absent $(\mathrm{G})$ whereas $\beta \mathrm{Gal}$ expression was seen ectopically in both $h$ th and exd knockdown ocelli where ocellar PRs were marked by Rh1 (red) $(H, I)$.

Figure 3: Expression of $\boldsymbol{r h 1}$ and $\boldsymbol{r h} 2$ reporter constructs in ocelli. (A) Schematic representation of the rh2 minimal promoter used for making GFP reporter constructs ranging from positions -293 to +55 of the transcription start $(+1$, arrow). The wildtype promoter (rh2-GFP; in the top) contains three potential Hth binding sites (green) with their corresponding sequences shown. In the hth mutant promoter (rh2(hth mut)-GFP;

511 in the bottom), the mutated sequences are shown with altered residues depicted in red. $(\mathrm{B}, \mathrm{C})$ Expression of the wildtype rh2-GFP and mutant $r$ 2(hth mut)-GFP reporter lines (green) in ocelli stained with antibodies against GFP (green), Rh2 (red) and Rh1

514 (blue). Wildtype rh2-GFP (marked by anti-GFP) is expressed in PRs of ocelli (marked by anti-Rh2) staining the entire rhabdomeres (B) whereas the mutant rh2(hth-mut)-

516 GFP (marked by anti-GFP) is not expressed in ocelli (C). (D) Schematic representation 517 of the rh1 minimal promoter used for making the GFP reporter constructs ranging from positions -247 to +73 of the transcription start $(+1$, arrow). The wildtype promoter (top) contains two potential Hth binding sites (green; with its corresponding sequence shown). In the hth mutant promoter (bottom), the mutated sequences are shown with

521 the altered residues depicted in red. (E, F) Expression of the wildtype rh1-GFP and 
522

523

524

525

526

527

528

529

530

531

532

533

534

535

536

537

538

539

540

541

542

543

544

545

546

mutant $r h 1$ (hth mut)-GFP reporter lines in ocelli stained with antibodies against GFP (green) Rh2 (red) and Rh1 (blue). (E) Like Rh1, the rh1-GFP reporter is not expressed in the PRs of ocelli expressing only Rh2. (F) The mutant $r h 1$ (hth-mut)-GFP reporter line is expressed in PRs of ocelli.

Figure 4: Hth misexpression phenotype in the retina. (A) Antibody staining to show expression of $\mathrm{Rh} 2$ and $\mathrm{Rh} 1$ in the wildtype retina. Rh2 (green) is normally not expressed in the retina $\left(A^{\prime}\right)$ whereas Rh1 (blue) is expressed in the outer PRs of retina (A"). PRs of the retina were stained with phalloidin (red). (B) Expression of Rh1 and Rh2 in the hth overexpression retina by IGMR-Gal4. Rh2 expression (green) can now be ectopically seen in the outer PRs of retina (B') whereas Rh1 expression (blue) remained unaffected (B").

Figure 5: Hth overexpression clones in the retina. (A, B, C, D) Antibody staining to mark $h$ th overexpression in clones (green) in the retina generated by the flip-out technique. (A, $\left.A^{\prime}\right)$ Antibody staining against GFP to identify inner PR clones of the retina, also co-stained by Rh2 (blue) and Phalloidin (red). Rh2 expression was not induced ( $\left.A^{\prime \prime}\right)$ when clones were generated in the inner PRs. (B, B') Antibody staining against GFP to identify outer PR clones of the retina, also co-stained by Rh2 (blue) and Phalloidin (red). When clones were generated in the outer PRs, Rh2 expression was induced (B'). White arrows marked inner and outer PR clones (A, A', B, B') and their corresponding Rh2 expression. (C, C', D, D') Antibody staining against GFP to identify inner and outer PR clones of the retina, also co-stained by Rh1 (blue) and Phalloidin (red). When clones were either induced in the inner PRs (C, C') or outer PRs (D, D') of the retina, Rh1 expression is unaffected (C', D'). 
548 Figure 6: Knockdown screening of Hth interactors in the ocelli. (A) Expression of

549 Rh1 (red) and Rh2 (green) in the wildtype ocelli. Ocellar PRs normally expresses Rh2 550 and Rh1 is usually absent. Knockdown of camta (B), Iola (C) and $d v e(\mathrm{D})$ by IGMR-

551 Gal4 showed loss of Rh2 expression (green) (previously found in [37]. However, Rh1 expression (red) were not ectopically induced in these knockdowns in the ocelli. Knockdown of scrib (E) and ets65a (F) showed a gain of Rh1 expression (red) in the ocelli whereas Rh2 expression (green) seemed to be unchanged in ocellar PRs.

Figure 7: Model for the binary Rhodopsin switch in the ocelli. Schematic representation showing origin of ocelli and compound eye from an ancestral eye in insects around 500 million years ago. We showed that Rh2 is normally expressed in the ocelli and gets independently regulated by Hazy and $\mathrm{Hth}$. Hth regulates the binary Rhodopsin switch to promote Rh2 expression in ocelli while repressing $\mathrm{Rh} 1$. Overexpression of $h$ th is sufficient to promote Rh2 expression but only at the outer-

562 PRs of the retina.

Figure S1: Phylogenetic analysis of opsin ( $r h 1, r h 2$ and $r h 6)$ duplications in

dipterans. Phylogenetic tree by maximum likelihood method showed predicted $r h 1$,

566 rh2 and rh6 duplications in different dipteran species, such as Drosophila

567 melanogaster, Ceratitis capitata, Musca domestica, Glossina palpalis, Lucilia cuprina,

568 Aedes aegypti and Anopheles gambiae. Opsin paralog groups were named according

569 to Drosophila nomenclature. Amino acid sequences of Rhodopsins were used for 570 making the phylogenetic tree and these sequences were available either in Feuda lab

571 on bitbucket (https://bitbucket.org/Feuda-lab/opsin diptera/src/master/) [30] or in 
$572 \mathrm{NCBI}$ (National Center for Biotechnology Information). The Phylogenetic tree was

573 made (by using MUSCLE online tool phylogeny.fr) and Rh5 sequence from Drosophila

574 were taken as an outgroup.

575

576 Figure S2: Independent roles of Hazy and Hth to regulate Rh2 expression in

ocelli. (A) Overexpression of $r h 1$ in all PRs of ocelli by using pan-photoreceptor IGMR-

578 Gal4. Antibody staining against Rh2 (green) and Rh1 (red) were performed to show

579

580

581

582

583

584

585

586

587

588

589

590

591

592

593

594

595

that overexpression of $r h 1$ does not inhibit Rh2 expression in ocelli. (B) Antibody staining to show expression of Rh2 (green) and Rh1 (red) in hazy/- null mutant ocelli.

Rh2 expression is lost is absent in hazy/- mutants but they don't show ectopic Rh1 expression in ocelli. (C) Antibody staining to show Hth expression (blue) in hazy/mutant ocelli, also marked by Chp (green). Hth expression is not affected in ocellar PRs in hazy/- mutants. (D) Antibody staining to show Hazy expression (blue) in hth knockdown ocelli. Ocelli in hth knockdown is marked by staining against Rh1 (red). Hazy is expressed during the Rhodopsin switch in hth knockdown ocelli.

Figure S5: Knockdown of Hth does not alter ocellar PR cell fate. (A, B) Antibody staining against Svp (green) in the wildtype and in hth knockdown ocelli. Svp is normally expressed in R3/R4 and R1/R6 pairs of the developing retinal PRs [36] and not in wildtype ocelli (A). During the Rhodopsin switch by $h$ th knockdown in ocelli, Svp is still not expressed in ocellar PRs (B). (C, D) Antibody staining against BarH1 (green) in the wildtype and in hth knockdown ocelli. BarH1 is normally expressed in R1/R6 pair of the developing retina [35] and not in wildtype ocelli (C). Upon Rhodopsin switch by $h$ th knockdown, BarH1 is still not expressed in the ocellar PRs (D). Ocellar PRs are 
marked by antibody staining against Elav (blue, in A and B) and Chp (red, in C and

597 D).

598

Figure S4: Alignment of the Rhodopsin 1 promoters of 12 Drosophila species.

600

601

602

603

604

605

606

607

608

609

610

611

612

613

614

615

616

617

618

619

620

Conserved residues are depicted on a grey background. For the reporter constructs we cloned a $320 \mathrm{bp}$ fragment ranging from nucleotides 2 to 320 of the $D$. melanogaster sequence in front of GFP. The two potential Hth binding sites are outlined in red. The RCSI is outlined in blue. The first Rh1 exon of $D$. melanogaster is outlined in black. In all twelve species the translation start (Met, arrow) is directly followed by an intron. Species used: D. melanogaster (Dmel), D. simulans (Dsim), D. sechellia (Dsec), D. yakuba (Dyak), D. erecta (Dere), D. ananassae (Dana), D. persimilis (Dper), D. pseudoobscura (Dpse), D. wilistoni (Dwil), D. grimshawi (Dgri), D. mojavensis (Dmoj), D. virilis (Dvir).

\section{Figure S5: Alignment of the Rhodopsin 2 promoters of 12 Drosophila species.}

Conserved residues are depicted on a grey background. For the reporter constructs we cloned the $348 \mathrm{bp}$ fragment of $D$. melanogaster from the endogenous Sal I restriction site (underlined in green) to the last nucleotide before the translation Start (Met, arrow) in front of GFP. This promoter sequence partially overlaps the last exon of the neighbouring gene CG14297. The three potential Hth binding sites are outlined in red. The first site is located within the coding sequence of CG14297. The second site which is only conserved within the melanogaster group (top five species) is located at the stop codon (asterisk) of CG14297, and the last site is located at the end of the 3'UTR. The RSCI (outlined in blue) is located within the 80 bp sequence between the end of the CG14297 3'UTR and the transcription start of Rhosopsin 2. The exons of 
D. melanogaster are outlined in black. Species used: $D$. melanogaster (Dmel), $D$.

D. mojavensis (Dmoj), D. grimshawi (Dgri), D. virilis (Dvir).

Figure S6: A table for knockdown mini-screening candidates in the ocelli. The

table consists of candidates either known to have interactions with Hth (information

Mishra et al., 2016). For knockdown screening, flies were ordered from Bloomington's

Drosophila stock center and the stock numbers are listed in the table.

\section{References:}

1. von Salvini-Plawen L, Mayr E. On the Evolution of Photorecptors and Eyes:

2. Quiring R, Walldorf U, Kloter U, Gehring WJ. Homology of the eyeless gene of Drosophila to the Small eye gene in mice and Aniridia in humans. Science. 1994;265(5173):785-9. Epub 1994/08/05. doi: 10.1126/science.7914031. PubMed PMID: 7914031.

3. Gehring WJ. The genetic control of eye development and its implications for the evolution of the various eye-types. Int J Dev Biol. 2002;46(1):65-73. Epub 2002/03/21. PubMed PMID: 11902689.

642

643

4. Kumar JP. Signalling pathways in Drosophila and vertebrate retinal development. Nat Rev Genet. 2001;2(11):846-57. Epub 2001/11/21. doi: 10.1038/35098564. PubMed PMID: 11715040. Science. 1994;265(5173):742-3. Epub 1994/08/05. doi: 10.1126/science.8047881. PubMed PMID: 8047881.

6. Wolff TaR, D.F. Pattern formation in the Drosophila retina. In: The Development of Drosophila melanogaster Cold Spring Harbor Laboratory Press. 1993;2:1277-325. 7. Yoon CS, Hirosawa K, Suzuki E. Studies on the structure of ocellar photoreceptor cells of Drosophila melanogaster with special reference to subrhabdomeric cisternae. Cell Tissue Res. 1996;284(1):77-85. Epub 1996/04/01. doi: 10.1007/s004410050568. PubMed PMID: 8601298.

654 8. Stark WS, Sapp R, Carlson SD. Ultrastructure of the ocellar visual system in normal and mutant Drosophila melanogaster. J Neurogenet. 1989;5(2):127-53. Epub 1989/05/01. doi: 10.3109/01677068909066203. PubMed PMID: 2500507. developmental corollaries of insect eye primordium evolution. Dev Biol. 
2006;299(2):310-29. Epub 2006/09/16. doi: 10.1016/j.ydbio.2006.08.027. PubMed PMID: 16973149.

10. Waloszek D. The 'Orsten' window - a three-dimensionally preserved Upper Cambrian meiofauna and its contribution to our understanding of the evolution of Arthropoda. Paleontological Research. 2003;7(1):71-88, 18.

11. Henze MJ, Dannenhauer K, Kohler M, Labhart T, Gesemann M. Opsin evolution and expression in arthropod compound eyes and ocelli: insights from the cricket Gryllus bimaculatus. BMC Evol Biol. 2012;12:163. Epub 2012/09/01. doi: 10.1186/1471-2148-12-163. PubMed PMID: 22935102; PubMed Central PMCID: PMCPMC3502269.

12. Friedrich M. Opsins and cell fate in the Drosophila Bolwig organ: tricky lessons in homology inference. Bioessays. 2008;30(10):980-93. Epub 2008/09/19. doi: 10.1002/bies.20803. PubMed PMID: 18800378.

13. Chou W-H, Hall KJ, Wilson DB, Wideman CL, Townson SM, Chadwell LV, et al. Identification of a novel Drosophila opsin reveals specific patterning of the $\mathrm{R} 7$ and R8 photoreceptor cells. Neuron. 1996;17(6):1101-15.

14. Huber A, Schulz S, Bentrop J, Groell C, Wolfrum U, Paulsen R. Molecular cloning of Drosophila Rh6 rhodopsin: the visual pigment of a subset of $\mathrm{R} 8$ photoreceptor cells. FEBS Lett. 1997;406(1-2):6-10. Epub 1997/04/07. doi: 10.1016/s0014-5793(97)00210-x. PubMed PMID: 9109375.

15. Papatsenko D, Sheng G, Desplan C. A new rhodopsin in R8 photoreceptors of Drosophila: evidence for coordinate expression with Rh3 in R7 cells. Development. 1997;124(9):1665-73. Epub 1997/05/01. PubMed PMID: 9165115.

16. Hardie RC. Functional organization of the fly retina. Progress in sensory physiology: Springer; 1985. p. 1-79.

17. O'Tousa JE, Baehr W, Martin RL, Hirsh J, Pak WL, Applebury ML. The Drosophila ninaE gene encodes an opsin. Cell. 1985;40(4):839-50. Epub 1985/04/01. doi: 10.1016/0092-8674(85)90343-5. PubMed PMID: 2985266.

18. Zuker CS, Cowman AF, Rubin GM. Isolation and structure of a rhodopsin gene from D. melanogaster. Cell. 1985;40(4):851-8. Epub 1985/04/01. doi: 10.1016/00928674(85)90344-7. PubMed PMID: 2580638.

19. Feiler R, Harris WA, Kirschfeld K, Wehrhahn C, Zuker CS. Targeted misexpression of a Drosophila opsin gene leads to altered visual function. Nature. 1988;333(6175):737-41. Epub 1988/06/23. doi: 10.1038/333737a0. PubMed PMID: 2455230.

20. Mismer D, Michael WM, Laverty TR, Rubin GM. Analysis of the promoter of the Rh2 opsin gene in Drosophila melanogaster. Genetics. 1988;120(1):173-80. Epub 1988/09/01. PubMed PMID: 2975615; PubMed Central PMCID: PMCPMC1203488.

21. Pollock JA, Benzer S. Transcript localization of four opsin genes in the three visual organs of Drosophila; RH2 is ocellus specific. Nature. 1988;333(6175):779-82. Epub 1988/06/23. doi: 10.1038/333779a0. PubMed PMID: 2968518.

22. Krapp HG. Ocelli. Curr Biol. 2009;19(11):R435-7. Epub 2009/06/12. doi: 10.1016/j.cub.2009.03.034. PubMed PMID: 19515345.

23. Stange G, Stowe S, Chahl J, Massaro A. Anisotropic imaging in the dragonfly median ocellus: a matched filter for horizon detection. Journal of Comparative Physiology A. 2002;188(6):455-67.

24. Bao R, Friedrich M. Molecular evolution of the Drosophila retinome: exceptional gene gain in the higher Diptera. Mol Biol Evol. 2009;26(6):1273-87. Epub 2009/03/03. doi: 10.1093/molbev/msp039. PubMed PMID: 19252076. 
25. Wernet MF, Labhart T, Baumann F, Mazzoni EO, Pichaud F, Desplan C. Homothorax switches function of Drosophila photoreceptors from color to polarized light sensors. Cell. 2003;115(3):267-79. Epub 2003/11/26. doi: 10.1016/s00928674(03)00848-1. PubMed PMID: 14636555.

26. Gao S, Takemura SY, Ting CY, Huang S, Lu Z, Luan H, et al. The neural substrate of spectral preference in Drosophila. Neuron. 2008;60(2):328-42. Epub 2008/10/30. doi: 10.1016/j.neuron.2008.08.010. PubMed PMID: 18957224; PubMed Central PMCID: PMCPMC2665173.

27. Yamaguchi S, Desplan C, Heisenberg M. Contribution of photoreceptor subtypes to spectral wavelength preference in Drosophila. Proc Natl Acad Sci U S A. 2010;107(12):5634-9. Epub 2010/03/10. doi: 10.1073/pnas.0809398107. PubMed PMID: 20212139; PubMed Central PMCID: PMCPMC2851746.

28. Velarde RA, Sauer CD, Walden KK, Fahrbach SE, Robertson HM. Pteropsin: a vertebrate-like non-visual opsin expressed in the honey bee brain. Insect Biochem Mol Biol. 2005;35(12):1367-77. Epub 2005/11/18. doi: 10.1016/j.ibmb.2005.09.001. PubMed PMID: 16291092.

29. Dereeper A, Guignon V, Blanc G, Audic S, Buffet S, Chevenet F, et al. Phylogeny.fr: robust phylogenetic analysis for the non-specialist. Nucleic Acids Res. 2008;36(Web Server issue):W465-9. Epub 2008/04/22. doi: 10.1093/nar/gkn180. PubMed PMID: 18424797; PubMed Central PMCID: PMCPMC2447785.

30. Feuda R, Goulty M, Zadra N, Gasparetti T, Rosato E, Segata N, et al. The diverging evolutionary history of opsin genes in Diptera. bioRxiv. 2020:2020.06.29.177931. doi: 10.1101/2020.06.29.177931.

31. Wernet MF, Desplan C. Homothorax and Extradenticle alter the transcription factor network in Drosophila ommatidia at the dorsal rim of the retina. Development. 2014;141(4):918-28. Epub 2014/02/06. doi: 10.1242/dev.103127. PubMed PMID: 24496628; PubMed Central PMCID: PMCPMC3912834.

32. Pai CY, Kuo TS, Jaw TJ, Kurant E, Chen CT, Bessarab DA, et al. The Homothorax homeoprotein activates the nuclear localization of another homeoprotein, extradenticle, and suppresses eye development in Drosophila. Genes Dev. 1998;12(3):435-46. Epub 1998/02/28. doi: 10.1101/gad.12.3.435. PubMed PMID: 9450936; PubMed Central PMCID: PMCPMC316489.

33. Rieckhof GE, Casares F, Ryoo HD, Abu-Shaar M, Mann RS. Nuclear translocation of extradenticle requires homothorax, which encodes an extradenticlerelated homeodomain protein. Cell. 1997;91(2):171-83. Epub 1997/11/05. doi: 10.1016/s0092-8674(00)80400-6. PubMed PMID: 9346235.

34. Vasiliauskas D, Mazzoni EO, Sprecher SG, Brodetskiy K, Johnston RJ, Jr., Lidder $\mathrm{P}$, et al. Feedback from rhodopsin controls rhodopsin exclusion in Drosophila photoreceptors. Nature. 2011;479(7371):108-12. Epub 2011/10/11. doi: 10.1038/nature10451. PubMed PMID: 21983964; PubMed Central PMCID: PMCPMC3208777.

35. Higashijima S, Kojima T, Michiue T, Ishimaru S, Emori Y, Saigo K. Dual Bar homeo box genes of Drosophila required in two photoreceptor cells, R1 and R6, and primary pigment cells for normal eye development. Genes Dev. 1992;6(1):50-60. Epub 1992/01/01. doi: 10.1101/gad.6.1.50. PubMed PMID: 1346120.

36. Mlodzik M, Hiromi Y, Weber U, Goodman CS, Rubin GM. The Drosophila seven-up gene, a member of the steroid receptor gene superfamily, controls photoreceptor cell fates. Cell. 1990;60(2):211-24. Epub 1990/01/26. doi: 10.1016/0092-8674(90)90737-y. PubMed PMID: 2105166. 
37. Mishra AK, Bargmann BOR, Tsachaki M, Fritsch C, Sprecher SG. Functional genomics identifies regulators of the phototransduction machinery in the Drosophila larval eye and adult ocelli. Dev Biol. 2016;410(2):164-77. Epub 2016/01/16. doi: 10.1016/j.ydbio.2015.12.026. PubMed PMID: 26769100.

38. Mishra M, Oke A, Lebel C, McDonald EC, Plummer Z, Cook TA, et al. Pph13 and orthodenticle define a dual regulatory pathway for photoreceptor cell morphogenesis and function. Development. 2010;137(17):2895-904. Epub 2010/07/30. doi: 10.1242/dev.051722. PubMed PMID: 20667913; PubMed Central PMCID: PMCPMC2938920.

39. Papatsenko D, Nazina A, Desplan C. A conserved regulatory element present in all Drosophila rhodopsin genes mediates Pax6 functions and participates in the finetuning of cell-specific expression. Mech Dev. 2001;101(1-2):143-53. Epub 2001/03/07. doi: 10.1016/s0925-4773(00)00581-5. PubMed PMID: 11231067.

40. Struhl G, Basler K. Organizing activity of wingless protein in Drosophila. Cell. 1993;72(4):527-40.

41. Bilder D, Li M, Perrimon N. Cooperative regulation of cell polarity and growth by Drosophila tumor suppressors. Science. 2000;289(5476):113-6. Epub 2000/07/07. doi: 10.1126/science.289.5476.113. PubMed PMID: 10884224.

42. Bilder D, Perrimon N. Localization of apical epithelial determinants by the basolateral PDZ protein Scribble. Nature. 2000;403(6770):676-80. Epub 2000/02/25. doi: 10.1038/35001108. PubMed PMID: 10688207.

43. Chen T, Bunting M, Karim FD, Thummel CS. Isolation and characterization of five Drosophila genes that encode an ets-related DNA binding domain. Dev Biol. 1992;151(1):176-91. Epub 1992/05/01. doi: 10.1016/0012-1606(92)90225-6. PubMed PMID: 1577186.

44. Hsu T, Schulz RA. Sequence and functional properties of Ets genes in the model organism Drosophila. Oncogene. 2000;19(55):6409-16. Epub 2001/02/15. doi: 10.1038/sj.onc.1204033. PubMed PMID: 11175357.

45. Brockmann A, Domínguez-Cejudo MA, Amore G, Casares F. Regulation of ocellar specification and size by twin of eyeless and homothorax. Dev Dyn. 2011;240(1):75-85. Epub 2010/11/26. doi: 10.1002/dvdy.22494. PubMed PMID: 21104743.

46. $\mathrm{Hu} K G$, Reichert $\mathrm{H}$, Stark WS. Electrophysiological characterization ofDrosophila ocelli. Journal of comparative physiology. 1978;126(1):15-24.

47. Briscoe AD. Six opsins from the butterfly Papilio glaucus: molecular phylogenetic evidence for paralogous origins of red-sensitive visual pigments in insects. J Mol Evol. 2000;51(2):110-21. Epub 2000/08/19. doi: 10.1007/s002390010071. PubMed PMID: 10948267.

48. Briscoe AD, Chittka L. The evolution of color vision in insects. Annu Rev Entomol. 2001;46:471-510. Epub 2000/12/09. doi: 10.1146/annurev.ento.46.1.471. PubMed PMID: 11112177.

49. Gebelein B, McKay DJ, Mann RS. Direct integration of Hox and segmentation gene inputs during Drosophila development. Nature. 2004;431(7009):653-9. Epub 2004/10/08. doi: 10.1038/nature02946. PubMed PMID: 15470419.

50. Ryoo HD, Marty T, Casares F, Affolter M, Mann RS. Regulation of Hox target genes by a DNA bound Homothorax/Hox/Extradenticle complex. Development. 1999;126(22):5137-48. Epub 1999/10/26. PubMed PMID: 10529430.

51. Kobayashi M, Fujioka M, Tolkunova EN, Deka D, Abu-Shaar M, Mann RS, et al. Engrailed cooperates with extradenticle and homothorax to repress target genes in Drosophila. Development. 2003;130(4):741-51. Epub 2002/12/31. doi: 
10.1242/dev.00289. PubMed PMID: 12506004; PubMed Central PMCID: PMCPMC2692026.

809 52. Inbal A, Halachmi N, Dibner C, Frank D, Salzberg A. Genetic evidence for the transcriptional-activating function of Homothorax during adult fly development. Development. 2001;128(18):3405-13. Epub 2001/09/22. PubMed PMID: 11566847. 53. Zelhof AC, Koundakjian E, Scully AL, Hardy RW, Pounds L. Mutation of the photoreceptor specific homeodomain gene Pph13 results in defects in phototransduction and rhabdomere morphogenesis. Development. 815 2003;130(18):4383-92. Epub 2003/08/06. doi: 10.1242/dev.00651. PubMed PMID: 81612900454.

817 54. Bischof J, Maeda RK, Hediger M, Karch F, Basler K. An optimized transgenesis 818 system for Drosophila using germ-line-specific phiC31 integrases. Proc Natl Acad Sci 819 U S A. 2007;104(9):3312-7. Epub 2007/03/16. doi: 10.1073/pnas.0611511104. PubMed PMID: 17360644; PubMed Central PMCID: PMCPMC1805588.

55. Hsiao HY, Johnston RJ, Jr., Jukam D, Vasiliauskas D, Desplan C, Rister J. Dissection and immunohistochemistry of larval, pupal and adult Drosophila retinas. J Vis Exp. 2012;(69):4347. Epub 2012/11/28. doi: 10.3791/4347. PubMed PMID: 23183823; PubMed Central PMCID: PMCPMC3523422. 56. Tahayato A, Sonneville R, Pichaud F, Wernet MF, Papatsenko D, Beaufils P, et al. Otd/Crx, a dual regulator for the specification of ommatidia subtypes in the Drosophila retina. Dev Cell. 2003;5(3):391-402. Epub 2003/09/12. doi: 10.1016/s1534-5807(03)00239-9. PubMed PMID: 12967559.

829 57. Noro B, Culi J, McKay DJ, Zhang W, Mann RS. Distinct functions of homeodomain-containing and homeodomain-less isoforms encoded by homothorax. Genes Dev. 2006;20(12):1636-50. Epub 2006/06/17. doi: 10.1101/gad.1412606. PubMed PMID: 16778079; PubMed Central PMCID: PMCPMC1482483.

58. Kanai MI, Okabe M, Hiromi Y. seven-up Controls switching of transcription factors that specify temporal identities of Drosophila neuroblasts. Dev Cell. 2005;8(2):203-13. Epub 2005/02/05. doi: 10.1016/j.devcel.2004.12.014. PubMed 836 PMID: 15691762.

837 59. Charlton-Perkins M, Whitaker SL, Fei Y, Xie B, Li-Kroeger D, Gebelein B, et al. 838 Prospero and Pax2 combinatorially control neural cell fate decisions by modulating 839 Ras- and Notch-dependent signaling. Neural Dev. 2011;6:20-. doi: 10.1186/17498104-6-20. PubMed PMID: 21539742. 
Figure 2

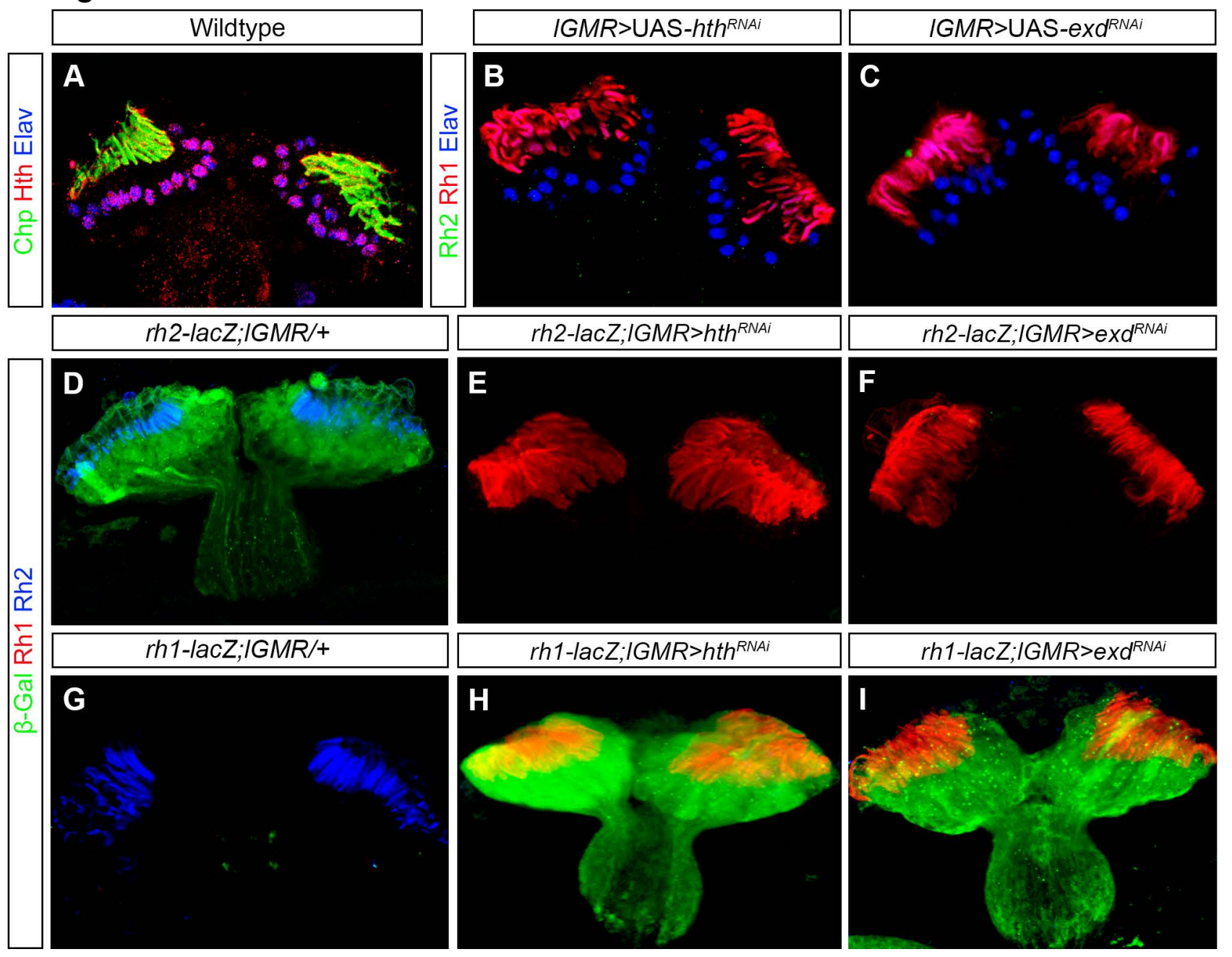


Figure 6

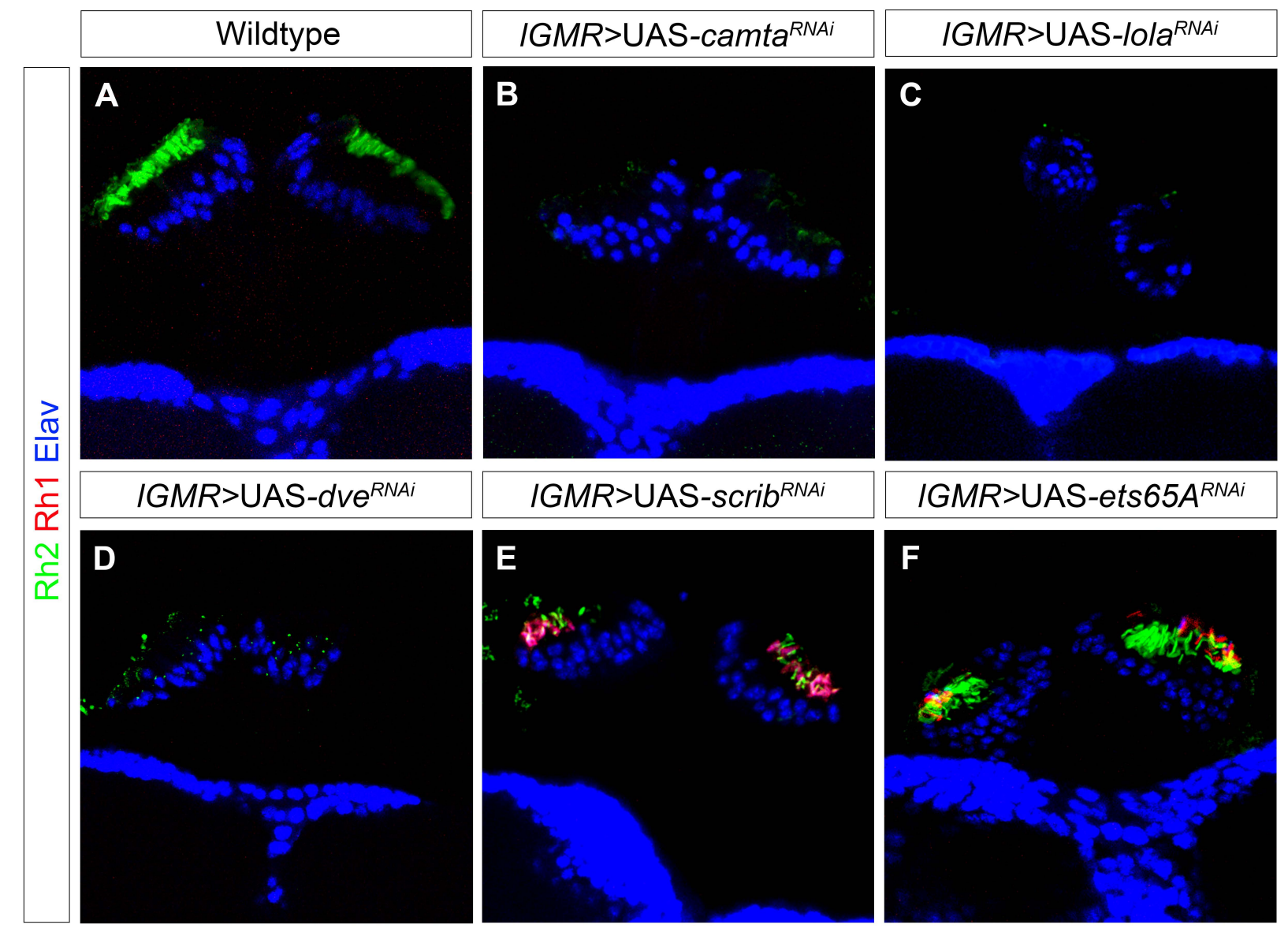


Figure 7

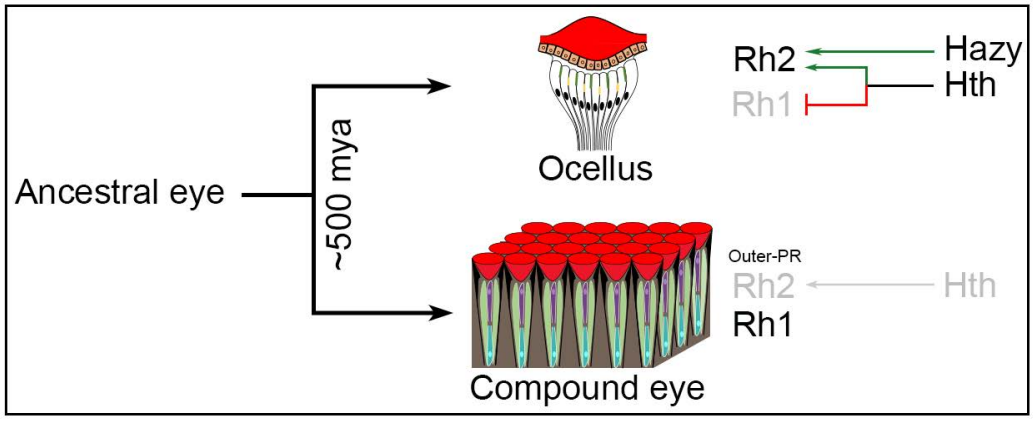

